\title{
Recommendations for the treatment of hepatitis C in 2017
}

\author{
Polish Group of Experts for HCV: Waldemar Halota, Robert Flisiak, Jacek Juszczyk, Piotr Małkowski,
} Małgorzata Pawłowska, Krzysztof Simon, Krzysztof Tomasiewicz

\begin{abstract}
The goals of treatment is to eliminate HCV infection, stop or reverse histological changes, reduce the risk of hepatocellular carcinoma development and transmission of the infection to other individuals. According to the recommendation of the Polish Group of Experts for HCV in 2017 all patients with chronic HCV infection should receive treatment, but it is not recommended in patients at high risk of short overall survival. If access to therapy is restricted, priority should be given to patients whose HCV infection can lead to an unfavourable outcome of the disease within a short time frame, particular to individuals with liver cirrhosis, rapidly progressing liver fibrosis, extrahepatic manifestations of HCV infection, chronic kidney diseases, patients before and after organ transplantation. Current recommendations of Polish Group of Experts for HCV provide guidelines to select optimal medication, assessment of liver fibrosis, treatment efficacy, dealing with resistance to direct acting antivirals, monitoring for hepatocellular carcinoma, management of HBV/HCV coinfection and drug interactions. It constains also advice on treatment of special patients populations such as renal failure, liver transplant and hepatic decompensation, as well as retreatment of patients which failed interferon free therapy. Moreover specific recommendations of management patients infected with different genotypes with currently reimbursed regimens or those expected to become available shortly in Poland are also included.
\end{abstract}

\section{Address for correspondence}

Prof. Waldemar Halota, Department of Infectious Diseases and Hepatology, Nicolaus Copernicus University Collegium Medicum, 12 Św. Floriana St., 85-030 Bydgoszcz, phone: +52 32556 05, e-mail: kikchzak@cm.umk.pl

\section{Introduction}

Diseases of hepatitis C virus (HCV) aetiology are rarely diagnosed on the basis of the clinical picture, since their course is usually asymptomatic or only mildly symptomatic for many years. Consequently, diagnosis is frequently preceded by an incidental detection of laboratory markers indicative of $\mathrm{HCV}$ infection. In recent years, anti-HCV antibodies have been identified in $0.9-1.9 \%$ of Poland's inhabitants, depending on the study population and the methodology applied. The studies have consistently confirmed the presence of HCV-RNA in the blood, recognized as an indicator of active infection, in $0.6 \%$ of the population. The figure corresponds to approximately 200,000 adult Poles who need urgent diagnosis and treatment. The number of patients diagnosed during the period of $\mathrm{HCV}$ therapy availability is estimated to be approximately 40,000 , which is equivalent to the detection rate of $20 \%$ [1-3]. Genotype (GT) $1 \mathrm{~b}$ is the most prevalent one in Poland (82\%). Other genotypes include GT3 (11.3\%), GT4 (3.5\%) and GT1a (3.2\%). Infections with genotypes 2, 5 and 6 may be diagnosed sporadically [4].

About $20-40 \%$ of acute infections tend to resolve spontaneously. Chronic HCV infection manifests itself after many years, and one in five patients develop advanced pathological changes in the liver including cirrhosis or hepatocellular carcinoma (HCC). HCV infection also induces a number of extrahepatic syndromes, most typically mixed cryoglobulinaemia, which gives rise to clinical manifestations in $5-25 \%$ of cases, and B-cell non-Hodgkin lymphoma (B-NHL) $[5,6]$.

All patients with chronic HCV infection should receive treatment. The sooner the therapy is initiated, the better the outcome and the lower the cost. The treatment is not recommended in patients at high risk of short overall survival. 
If access to therapy is restricted, priority should be given to the patients whose $\mathrm{HCV}$ infection, in the assessment of an infectious diseases specialist, can lead to an unfavourable outcome of the disease within a short time frame.

The above applies in particular to:

- liver cirrhosis (F4),

- rapidly progressing liver fibrosis (one-point increase during one year of follow-up in individuals with previously diagnosed fibrosis),

- extrahepatic manifestations of HCV infection,

- chronic kidney diseases,

- before and after organ transplantation.

The goals of treatment are to eliminate HCV infection and, consequently, to impede or reverse histological changes, reduce the risk of HCC development and transmission of the infection to other individuals [7].

\section{Acute HCV infection}

The only objective criterion in the diagnosis of acute hepatitis $\mathrm{C}$ (AHC) is the identification of AHC-associated laboratory markers (elevated alanine aminotransferase activity, presence of anti-HCV and/or HCV-RNA) in patients whose prior HCV tests were negative or in patients who had a documented exposure to $\mathrm{HCV}$ infection. In other cases, the diagnosis of AHC may be inconclusive. Importantly, while HCV-RNA is detectable as early as 1-3 weeks after infection, anti-HCV antibodies are not detected until 4-10 weeks. Following the onset of the first clinical manifestations, if they appear, anti-HCV antibodies are present in only $50-70 \%$ of infected patients, and it is only after three months that the proportion exceeds $90 \%$. Some patients do not develop anti-HCV antibodies at all. In such cases, the basis for diagnosing the infection is the presence of HCV-RNA in the blood.

Data confirming the efficacy of interferon-free therapy in hepatitis $\mathrm{C}$ are limited, however they show that the achieved SVR rates are at a level similar to that observed in chronic HCV [8]. In such situations the treatment should comply with rules governing the therapy of chronic infections. To avoid unnecessary therapy in patients who may eliminate the infection spontaneously, treatment may be initiated 24 weeks after HCV infection is diagnosed. However, in cases of acute HCV infection (recurrence) after liver transplantation, treatment should be initiated without such a delay.

\section{Chronic HCV infections}

The basic criterion for diagnosing chronic diseases of HCV aetiology is the presence of HCV-RNA (in blood serum, liver tissue or peripheral blood mononuclears) persisting for at least six months in a patient with markers of liver disease or an extrahepatic manifestation of the infection. HCV infection in the liver may lead to changes described as chronic hepatitis $\mathrm{C}$ and cirrhosis or hepatocellular carcinoma. HCV-infected patients diagnosed with cirrhosis do not need to wait six months for the initiation of therapy. The process of assessing eligibility for treatment should involve the determination of the viral genotype, and if genotype 1 is detected, also the determination of subgenotype (GT1a or GT1b) and evaluation of the stage of liver fibrosis. The course of the infection should be monitored by testing HCV-RNA with the use of techniques with the limit of detection $\leq 15 \mathrm{IU} / \mathrm{ml}$.

\section{General recommendations}

The therapeutic regimen must be selected on the basis of its current availability, efficacy and safety profile. Patients should be informed about the duration of therapy, potential adverse reactions associated with each drug, possible interactions with other drugs used in therapy, importance of adherence to the prescribed treatment regimen and rules for continuing or interrupting therapy.

\section{Recommended drugs}

Table 1 lists the majority of recommended drugs approved in any country worldwide, particularly by the EMA (European Medicines Agency) or FDA (Food and Drug Administration), as they are currently available or likely to become available on the Polish market in the near future. The use of drugs which are not listed in Table 1 is also acceptable, provided that they are approved according to their SPC [9].

\section{Assessment of liver fibrosis}

The degree of liver fibrosis should be assessed on a 5-point scale from 0 to 4 using a dynamic elastography technique offering the possibility to evaluate the stiffness of the liver tissue in $\mathrm{kPa}$ (SWE - share wave elastography, TE - transient elastography, ARFI - acoustic radiation force impulse), or liver biopsy. If coexisting liver diseases of a different aetiology are suspected, and the result of a non-invasive examination is inconsistent with the patient's clinical condition or discrepancies are shown between the results of various non-invasive tests, liver biopsy is recommended (unless contraindications to the procedure exist). In such cases biopsy results are regarded as conclusive [7]. If contraindications exist to liver biopsy and elastography, or if the test result is 
Table 1. Dosage regimens of drugs included in the Recommendations (drugs in respective groups are listed alphabetically)

\begin{tabular}{|c|c|c|c|}
\hline Drug category & Class & Drugs & Daily dosage \\
\hline \multirow[t]{11}{*}{ Direct acting antivirals (DAA) } & NS3 inhibitors (proteases) & Asunaprevir (ASV) & 200 mg/day in 2 doses \\
\hline & & Grazoprevir (GZR) & 100 mg/day in 1 dose* \\
\hline & & Paritaprevir (PTV) & $150 \mathrm{mg} /$ day in 1 dose $\mathrm{e}^{* *}$ \\
\hline & & Symeprevir (SMV) & 150 mg/day in 1 dose \\
\hline & NS5B inhibitors (polymerases) & Dasabuvir (DSV) & $500 \mathrm{mg} /$ day in 2 doses \\
\hline & & Sofosbuvir (SOF) & $400 \mathrm{mg} /$ day in 1 dose $\mathrm{e}^{* *}$ \\
\hline & NS5A inhibitors & Daclatasvir (DCV) & $60 \mathrm{mg} /$ day in 1 dose \\
\hline & & Elbasvir (EBR) & $50 \mathrm{mg} /$ day in 1 dose* \\
\hline & & Ledipasvir (LDV) & $90 \mathrm{mg} /$ day in 1 dose ${ }^{* * *}$ \\
\hline & & Ombitasvir (OBV) & $25 \mathrm{mg} /$ day in 1 dose** \\
\hline & & Velpatasvir (VEL) & 100 mg/day in 1 dose*** \\
\hline \multirow[t]{4}{*}{ Interferons } & Pegylated interferons $\alpha$ in children & PeglFN $\alpha-2 a^{\#}$ & $65-180 \mu \mathrm{g} / \mathrm{m}^{2} /$ week \\
\hline & & PeglFN $\alpha-2 b^{\# \#}$ & $60 \mu \mathrm{g} / \mathrm{m}^{2} /$ week \\
\hline & Pegylated interferons $\alpha$ in adults & PegIFN $\alpha-2 a$ & $180 \mu \mathrm{g} /$ week $^{\#}$ \\
\hline & & PegIFN $\alpha-2 b$ & $1.5 \mu \mathrm{g} / \mathrm{kg} /$ week \\
\hline \multirow[t]{3}{*}{ Others } & Ribavirin in adults & Ribavirin (RBV) & $1,000 \mathrm{mg}$ at body weight $<75 \mathrm{~kg}$ \\
\hline & & & $1,200 \mathrm{mg}$ at body weight $>75 \mathrm{~kg}$ \\
\hline & Ribavirin in children & Ribavirin (RBV) & 15 mg/kg \\
\hline
\end{tabular}

${ }^{*} G Z R$ and EBR are available in one tablet

**PTV and OBV are available in one tablet with ritonavir (r)

$* * *$ SOF is available alone or in one tablet with LDV or VEL

\#PegIFNo-2a in children: 65-180 $\mu \mathrm{g} /$ week depending on the body surface area

\#PegIFN $\alpha$-2b in children: $60 \mu \mathrm{g} / \mathrm{m}^{2} /$ week

non-assessable, treatment eligibility may be determined on the basis of results obtained in one of available serum tests. The simplest of them is APRI (aspartate aminotransferase/platelet ratio index), which indicates advanced liver fibrosis at values in the range of 1.0-2.0, and probable cirrhosis above 2.0 [10].

\section{Assessment of treatment efficacy}

Treatment may be considered effective if no HCVRNA is detected in blood 12 weeks after the completion of therapy, which corresponds to the achievement of sustained virological response (SVR12). The reliability of the result can be increased by repeating the test after another 12 weeks. In interferon-based therapy similar conclusions can be reached on the basis of results of HCV-RNA tests performed 24 weeks after the completion of therapy (SVR24).

The efficacy of therapy should be assessed by PCR methods which provide the detection level of $\leq 15$ IU/ $\mathrm{ml}[7]$.

\section{Resistance to DAA (direct acting antivirals)}

On account of the risk of selection of resistant variants (RASs - resistance associated substitutions) DAA monotherapy is unacceptable. Interferon-free therapy should combine between two and four NS3, NS5A and NS5B inhibitors, possibly in conjunction with RBV. RASs have the greatest practical significance for NS5A owing to the persistent nature of resistance and its widespread occurrence. There are as yet no established optimum retherapies for unsuccessfully treated patients in whom RASs are detected.

\section{Hepatocellular carcinoma (monitoring, DAA therapy)}

HCV-infected individuals, especially with coexisting cirrhosis, should be systemically monitored for the development of HCC by liver ultrasound and, if necessary, also by evaluating $\alpha$-fetoprotein (AFP) levels. Liver ultrasonography is mandatory prior to starting therapy, within 12 weeks after its completion, and at 24 -week intervals after that. The minimum duration of such a follow-up is 4 years, however it should be longer in patients with cirrhosis or a history of HCC [11].

Even though the evaluation of AFP concentration should not be applied for the early diagnosis of HCC, it may be useful for determining the prognosis of previously diagnosed cancer and for the monitoring of therapy administered to the patient.

If a cancer lesion is suspected, four-phase computed tomography (CT) scan with contrast or magnetic 
resonance imaging (MRI) with contrast is recommended. Contrast-enhanced ultrasonography, however, is not recommended for the routine diagnosis of HCC. Both ultrasound and CT/MRI scan should be performed by radiologists experienced in liver imaging.

The claims that DAA therapy increases the risk of hepatocarcinogenesis have not been proven, however HCC has been reported to occur during DAA treatment. There is no evidence to exclude the possibility that the reported cases involved the manifestation of hepatocellular carcinoma which started developing before the introduction of antiviral drugs [12-17]. The situation is different in HCV-infected patients with a history of HCC treatment (resection, thermoablation). The initiation of anti-HCV therapy is associated with the risk of relapse of liver cancer characterized by high dynamics of the disease. This is observed in particular in elderly men with advanced liver fibrosis in whom DAAs were introduced within 6 months after the treatment. Also in this case it is likely that therapy was initiated in patients with cancer recurrence. A good diagnostic criterion in these situations was an increase in AFP concentration [18-21]. Consequently, patients with a history of HCC treatment are a group in which cancer recurrence should be particularly carefully excluded (by CT, NMR, AFP) during a follow-up of at least six months. After the period, anti-HCV therapy may be started [22].

\section{HBV and HIV co-infections}

The therapy of HBV/HCV or HIV/HCV co-infection is the same as the treatment recommended for HCV monoinfection. It has recently been noted that DAA treatment in patients with HCV/HBV co-infection may cause life-threatening reactivation of $\mathrm{HBV}$ infection. Such cases have been recorded mainly in Asia, typically affecting patients between weeks 4 and 8 of therapy $[23,24]$.

In view of the above observations an HBsAg test is recommended and, as an addition, anti-HBc-total testing should be considered in every patient assessed for DAA therapy. Currently available data suggest that reactivation in $\mathrm{HBsAg}(-)$, anti-HBc-total $(+)$ patients is highly unlikely, however it cannot be ruled out. Individuals with presence of HBsAg or anti-HBc-total should be tested for HBV-DNA prior to the initiation of treatment. In the course of the therapy ALT levels should be monitored every two to four weeks in accordance with the following recommendations:

a) in cases where HBV-DNA is undetectable and ALT activity are normal prior to treatment, if the ALT activity rises above the upper limit of normal range during DAA therapy, HBV-DNA should be measured immediately and, without waiting for the result, treatment with a nucleoside analogue (entecavir) or a nucleotide analogue (tenofovir) should be initiated in parallel to DAA therapy;

b) in cases where HBV-DNA is undetectable, and ALT activity exceed the upper limit of normal range and fail to decrease during the first four weeks of DAA treatment, the HBV-DNA test should be repeated, and performed regularly until the end of therapy. If $\mathrm{HBV}$ viraemia is detected, the procedure to follow is outlined in item a);

c) in cases where HBV-DNA is detectable prior to treatment, one of the analogues listed above should be introduced a month before the initiation of DAA therapy;

d) in patients treated for HBV infection prior to the initiation of DAA the treatment should be sustained and DAA therapy should be initiated in parallel.

\section{Renal failure}

Patients with eGFR $\geq 30 \mathrm{ml} / \mathrm{min} / 1.73 \mathrm{~m}^{2}$ should receive treatment in line with general principles of HCV therapy. In GT1- or GT4-infected patients with severe renal impairment (eGFR $\left.<30 \mathrm{ml} / \mathrm{min} / 1.73 \mathrm{~m}^{2}\right)$, especially those receiving haemodialysis treatment, the optimum therapy is GZR/EBR or OBV/PTV/r + DSV. However, no optimum therapy is currently available for genotype 3-infected patients with renal impairment. The most beneficial therapeutic regimen is the combination of sofosbuvir and daclatasvir. RBV should be avoided, and renal function should be checked regularly, especially in patients receiving sofosbuvir.

\section{Liver transplantation}

The precondition for protecting transplanted liver from the relapse of HCV infection is the suppression of viraemia to undetectable levels at least a month prior to the transplantation procedure. Consequently, therapy should be initiated as early as possible after the patient's approval for liver transplantation. Early onset of therapy offers an opportunity to avoid liver transplantation in patients with the MELD score $\leq 20$.

Antiviral therapy in patients with advanced hepatic insufficiency (MELD > 20) should be preceded by the liver transplantation procedure. The above also applies to patients in situations where the expected pre-transplantation waiting period is too short to ensure effective suppression of HCV viraemia. In such cases patients require close monitoring after the transplantation pro- 
cedure in order to promptly detect a possible relapse of viraemia and, if it occurs, initiate interferon-free therapy within a month after HCV-RNA detection.

Patients undergoing liver transplantation during antiHCV therapy should continue treatment for 12 weeks post procedure. Before the treatment is started, potential drug interactions with DAAs should be considered to determine whether dosage adjustment or drug change may be needed [25-27].

The optimum treatment regimen to be used in patients after liver transplantation, regardless of the infection genotype, is SOF/VEL.

Alternative options in patients infected with $\mathrm{HCV}$ genotypes 1 and 4 are SOF/LDV $\pm \mathrm{RBV}$ or OBV/PTV/r $\pm \mathrm{RBV}$, genotype 2 is $\mathrm{SOF}+\mathrm{RBV}$ and genotype 3 is $\mathrm{SOF}+\mathrm{DCV} \pm \mathrm{RBV}[7,8,27-29]$. It is noted that a reduction in the dosage of immunosuppressive drugs may occasionally be needed.

\section{Patients with decompensated cirrhosis}

Therapy in patients with a history of hepatic encephalopathy, ascites, Child-Pugh scores B and C and in patients after liver transplantation should be conducted under careful monitoring in medical centres with experience in the treatment of patients with decompensated cirrhosis. The treatment centres should provide a possibility for immediate hospitalization and assessment of patient eligibility for liver transplantation. Patients with cirrhosis and Child-Pugh class $\mathrm{C}$ should be primarily recognized as eligible for liver transplantation. According to the SPCs, PTV/OBV/r are not indicated in liver failure class $\mathrm{B}$ and contraindicated in class $\mathrm{C}$, whereas GZR and EBR are contraindicated in both these cases. The risk of hepatic function deterioration secondary to $\mathrm{DAA}$ therapy with OBV/PTV/r $\pm \mathrm{DSV} \pm \mathrm{RBV}$ has been shown to be similar to the SOF/LDV but lower than in the SOF/SMV regimen $[27,30]$.

\section{DAA drug interactions}

Before HCV treatment is initiated, potential interactions with other drugs used by the patient must be assessed to determine their potential for affecting the efficacy, dosage or safety of treatment. If serious potential interactions exist, previously used drugs should be substituted for safe alternatives or another appropriate $\mathrm{HCV}$ treatment regimen should be considered. The above also applies to patients with renal failure, in whom sofosbuvir treatment may be contraindicated. Special attention should be given to immunosuppressive drugs which usually require dose reduction in DAA treatment; the exception is sofosbuvir. Most uncertain- ties about drug interactions can be resolved by checking the website at www.hep-druginteractions.org [26].

\section{Specific recommendations}

The basic criterion determining the therapeutic approach is HCV genotype. The therapeutic options in Table 2 which are recommended as first-line therapies are underlined. The therapeutic options proposed for patients after treatment failure are shown in Table 5.

\section{HCV genotype 1 infections}

The optimal therapy of GT1 infections in treatment-naive patients and after the failure of PegIFN $\alpha+$ $\mathrm{RBV}$ treatment or triple therapies with BOC or TVR is $\mathrm{OBV} / \mathrm{PTV} / \mathrm{r}+\mathrm{DSV}$ or SOF/LDV - in some cases requiring combination with RBV.

Other two therapeutic combinations are GZR/EBV $\pm \mathrm{RBV}$ and SOF/VEL $\pm \mathrm{RBV}$.

Treatment-naive GT1b-infected individuals without cirrhosis may also be considered for the ASV + DCV combination.

Ombitasvir/Paritaprevir/Ritonavir + Dasabuvir. Patients infected with HCV subgenotype $1 \mathrm{~b}$, regardless of previous treatment (also following unsuccessful triple interferon-based therapy) and the stage of fibrosis (also in cirrhosis) should receive OBV/PTV/r + DSV for 12 weeks without the need of adding RBV. The duration of treatment may be reduced to 8 weeks in patients with liver fibrosis stage F2 or lower.

In cases of infection with HCV subgenotype 1a, the regimen is supplemented with $\mathrm{RBV}$, and in patients with cirrhosis the duration of therapy is extended to 24 weeks.

The therapeutic management in patients infected with HCV of an unknown or inconclusive GT1 subgenotype or with mixed GT1a/1b infection should be the same as in patients infected with HCV genotype 1a. A 24-week OBV/PTV/r + DSV + RBV therapy should be initiated after liver transplantation, regardless of HCV subtype [30-32]. Real world experience (RWE) studies demonstrate the efficacy of the therapy especially in genotype $1 b$-infected patients, regardless of the stage of fibrosis (including patients with compensated cirrhosis).

Sofosbuvir/Ledipasvir. The SOF/LDV regimen in treatment-naive patients without cirrhosis should last 12 weeks, however it may be reduced to 8 weeks in genotype $1 \mathrm{~b}$-infected patients with liver fibrosis stage F2 or lower.

Patients with a history of treatment failure, with cirrhosis and after liver transplantation should receive 
Table 2. Recommended therapies and their duration depending on the viral genotype

\begin{tabular}{|c|c|c|c|c|c|c|c|}
\hline & GT1a & GT1b & GT2 & GT3 & GT4 & GT5 & GT6 \\
\hline OBV/PTV/r + DSV + RBV & 12-24 weeks & & & & & & \\
\hline OBV/PTV/r + DSV & & $\underline{8-12 \text { weeks }}$ & & & & & \\
\hline OBV/PTV/r \pm RBV & & & & & $\underline{12-24 \text { weeks }}$ & & \\
\hline ASV + DCV & & 24 weeks & & & & & \\
\hline SOF/LDV \pm RBV & $\underline{8-24 \text { weeks }}$ & $\underline{8-24 \text { weeks }}$ & & & $\underline{12-24 \text { weeks }}$ & $\underline{12-24 \text { weeks }}$ & $\underline{12-24 \text { weeks }}$ \\
\hline SOF + PeglFN $\alpha+$ RBV & & & & 12 weeks & 12 weeks & 12 weeks & 12 weeks \\
\hline $\mathrm{SOF}+\mathrm{RBV}$ & & & 12-24 weeks & 24 weeks & 24 weeks & 24 weeks & 24 weeks \\
\hline $\mathrm{SOF}+\mathrm{DCV}+\mathrm{RBV}$ & & & 24 weeks & 24 weeks & & & \\
\hline SOF/VEL \pm RBV & $\underline{12 \text { weeks }}$ & $\underline{12 \text { weeks }}$ & $\underline{12 \text { weeks }}$ & $\underline{12 \text { weeks }}$ & $\underline{12 \text { weeks }}$ & $\underline{12 \text { weeks }}$ & $\underline{12 \text { weeks }}$ \\
\hline $\mathrm{GZR} / \mathrm{EBR} \pm \mathrm{RBV}$ & 12-16 weeks & $\underline{12 \text { weeks }}$ & & & $\underline{12-16 \text { weeks }}$ & & \\
\hline PeglFN $\alpha+$ RBV$^{*}$ & 48 weeks & 48 weeks & 24 weeks & 24 weeks & 48 weeks & & \\
\hline
\end{tabular}

+ - combined therapy with drugs available as separate preparations

I - combined therapy with drugs available as one combined preparation

\pm - addition of RBV depending on indications; details are provided in the text

*Only recommended in children

SOF/LDV + RBV for 12 weeks. In cases where RBV may not be used, the period of treatment is extended to 24 weeks [32-34].

Asunaprevir + Daclatasvir. ASV + DCV is a regimen which may be considered in treatment-naive genotype $1 \mathrm{~b}$-infected patients without cirrhosis. The duration of therapy is 24 weeks. Importantly, the treatment is well tolerated by the elderly. The claim that the ASV + DCV combination leads to the selection of drug-resistant strains has not been confirmed in Poland as yet [35-37].

Grazoprevir + Elbasvir. GZR/EBR therapy in GT1-infected patients should last 12 weeks. In genotype 1a-infected patients with baseline viraemia $>800,000 \mathrm{IU} / \mathrm{ml}, \mathrm{GZR} / \mathrm{EBR}$ should be used in combination with RBV, and the period of treatment should be extended to 16 weeks. RBV should be added to the regimen in patients who have failed triple interferon-based treatment (with a protease inhibitor). The duration of GZR/EBR+RBV treatment should be extended to 16 weeks in GT1a-infected patients with NS5A-specific RASs [38].

Sofosbuvir/Velpatasvir. The therapy should be used for 12 weeks regardless of the stage of fibrosis and failure of previous treatment. Ribavirin may be considered as an addition to the therapeutic regimen in cases of decompensated cirrhosis [39].

Pegylated interferon $\alpha+$ Ribavirin. A 48-week PegIFNa-2b + RBV therapy is recommended in children over 3 years of age, and PegIFN $\alpha-2 a+$ RBV may be used in children over 5 years of age [40,41].

Children with advanced liver fibrosis (stages F3-F4) and sufficient body weight should be considered for the initiation of therapies prescribed for adults (subject to the consent of a competent ethics committee).

\section{HCV genotype 2 infection}

A 12-week SOF/VEL regimen is the therapy of choice regardless of the stage of fibrosis both in treatment-naive patients and for retherapy. Ribavirin is added to therapy in cases of decompensated cirrhosis [39]. An alternative therapeutic regimen is a 12-week course of SOF + RBV treatment which is successful in the majority of treatment-naive patients. The treatment is extended to 24 weeks in patients after liver transplantation and with high HCV viraemia or previously treated with PegIFN $\alpha+$ RBV [42].

If SOF $+\mathrm{RBV}$ is ineffective, a 12-week SOF/VEL regimen or a 24-week $\mathrm{SOF}+\mathrm{DCV}+\mathrm{RBV}$ regimen is recommended [7].

In children the recommended duration of treatment is 24 weeks: PegIFN $\alpha-2 b+$ RBV is used in children over 3 years of age, and PegIFN $\alpha-2 a+R B V-$ in children over 5 years of age $[40,41]$.

\section{HCV genotype 3 infection}

The optimum therapeutic option is a 12-week SOF/ VEL regimen, combined with RBV in patients with cirrhosis. A 12-week SOF + PegIFN + RBV treatment ensures an equally high efficacy, particularly in cirrhosis-free patients. The main limitations of the therapy, however, are contraindications and adverse events associated particularly with interferon use (Tables 3 and 4). In cases of interferon intolerance the doses of 
the drug may be reduced or treatment with the other two drugs may be continued for a total of 24 weeks. Patients with contraindications to interferon may be treated with SOF + RBV alone for 24 weeks [42, 43].

Patients failing therapy with SOF + RBV \pm PegIFN $\alpha$ should receive a 12 -week therapy with SOF/ $\mathrm{VEL} \pm \mathrm{RBV}$. Alternatively, a 24-week SOF + DCV + $\mathrm{RBV}$ regimen may be considered.

In children the recommended duration of treatment is 24 weeks: PegIFN $\alpha-2 b+R B V$ is used in children over 3 years of age, and PegIFN $\alpha-2 a+R B V-$ in children over 5 years of age $[40,41]$.

\section{HCV genotype 4 infection}

The optimum therapy in patients infected with genotype 4, both treatment-naive and with a history of unsuccessful PegIFN $\alpha+$ RBV treatment, is OBV/PTVr $+\mathrm{RBV}$. Other therapeutic options which, however, are not currently reimbursed in Poland include SOF/LDV $\pm \mathrm{RBV}, \mathrm{GZR} / \mathrm{EBR} \pm \mathrm{RBV}$ and SOF/VEL $\pm \mathrm{RBV}$.

Ombitasvir/Paritaprevir/Ritonavir. OBV/PTV/r should be used in combination with RBV for 12 weeks regardless of the degree of liver fibrosis. In patients after liver transplantation OBV/PTVr + RBV should be continued for 24 weeks [30, 31].

Sofosbuvir/Ledipasvir. The therapy lasts 12 weeks in treatment-naive cirrhosis-free patients. In patients with cirrhosis, with history of treatment failure or after liver transplantation SOF/LDV + RBV is used for 12 weeks, and if there are contraindications to ribavirin, the duration of therapy should be extended to 24 weeks [32].

Sofosbuvir/Velpatasvir. Regardless of the stage of liver fibrosis the drugs should be used for 12 weeks. RBV should be added to therapy in patients with decompensated cirrhosis [39].

Grazoprevir+Elbasvir. Therapy with GZR/EBR lasts 12 weeks, however in patients previously unsuccessfully treated with IFN + RBV it is extended to 16 weeks, and ribavirin is added to the regimen [38].

$P e g I F N \alpha+R B V$. In children the recommended duration of treatment is 48 weeks: PegIFN $\alpha-2 b+R B V$ is used in children over 3 years of age, and PegIFN $\alpha-2 a$ $+\mathrm{RBV}$ - in children over 5 years of age $[40,41]$.

\section{Infection with HCV genotypes 5 and 6}

Sofosbuvir/Ledipasvir. Treatment-naive, cirrhosisfree patients should receive therapy for 12 weeks. The option of shortening the duration of treatment to eight weeks has not been confirmed yet. Patients who are eligible for retherapy, with cirrhosis or post liver
Table 3. Contraindications to interferon $\alpha$ therapy

- History of hypersensitivity to interferons or any of the excipients

- Decompensated cirrhosis

- Hepatitis or another disease of autoimmune aetiology

- Status post transplantation of liver or any other organ

- Patients approved for liver transplantation

- Pregnancy

- Severe (especially unstable) heart disease

- Generalized atherosclerosis

- Chronic respiratory failure

- Metabolic syndrome and difficult-to-treat diabetes, following consultation with an endocrinologist

- Depression, suicidal ideation or attempts documented by a psychiatric evaluation

- Thyroid diseases involving abnormal TSH levels

- Anaemia

- Thrombocytopaenia < 90,000/ $\mu 1$

- Absolute neutrophil count $<1,500 / \mu l$

Table 4. Criteria of interferon intolerance

- Hypersensitivity to interferon or any of the excipients

- Autoimmunization disease

- Exacerbation of a previously existing comorbidity

- Decrease in body weight by more than $20 \%$ relative to the baseline

- Depression, suicidal ideation or attempts

- Thyroid dysfunction

- Haemoglobin concentration $<8.5 \mathrm{mg} \%$

- Thrombocytopaenia $<50,000 / \mu 1$

- Absolute neutrophil count $<500 / \mu 1$

transplantation should additionally receive ribavirin or their treatment should be extended to 24 weeks [32, 33].

Sofosbuvir/Velpatasvir. The treatment should last 12 weeks regardless of the stage of liver fibrosis, both in treatment-naive patients and in individuals eligible for retreatment. Ribavirin should be considered as an addition to therapy in patients with decompensated liver function [39].

An alternative therapeutic option is the SOF + PegIFN $\alpha+$ RBV combination used for 12 weeks. In cases of intolerance leading to interferon discontinuation $\mathrm{SOF}+\mathrm{RBV}$ should be continued for 24 weeks. Both drugs are used for 24 weeks in patients with contraindications to IFN and after liver transplantation [40, 41].

\section{Retherapy of HCV infections}

Table 5 lists proposed therapeutic options in the treatment of chronic liver diseases of HCV aetiology in patients after previous treatment failure. Testing for RASs is not required in routine clinical practice in such cases.

Patients with advanced liver fibrosis should be prioritized for retherapy. 
Table 5. Therapeutic options recommended in retherapy of HCV infections (alphabetically)

\begin{tabular}{|c|c|c|}
\hline Genotype & Ineffective therapy & Proposed retherapy \\
\hline \multirow[b]{2}{*}{1} & $\begin{array}{l}\text { BOC + PeglFN + RBV } \\
\text { PeglFN + RBV } \\
\text { SMV + PegIFN + RBV } \\
\text { TVR + PegIFN + RBV }\end{array}$ & $\begin{array}{l}\mathrm{GZR} / \mathrm{EBR} \pm \mathrm{RBV} \\
\mathrm{SOF} / \mathrm{LDV} \pm \mathrm{RBV} \\
\mathrm{OBV} / \mathrm{PTV} / \mathrm{r}+\mathrm{DSV} \pm \mathrm{RBV} \\
\mathrm{VEL} / \mathrm{SOF}\end{array}$ \\
\hline & $\begin{array}{l}A S V+D C V \\
G Z R / E B R \pm R B V \\
L D V / S O F \pm R B V \\
O B V / P T V / r+D S V \pm R B V \\
\text { VEL/SOF }\end{array}$ & $\begin{array}{l}\text { F0-F3: more effective proposed therapies are awaited } \\
\text { F4: } \\
\text { SOF + GZR/EBR + RBV } \\
\text { SOF + OBV/PTV/r + DSV } \pm \text { RBV } \\
\text { SOF + SMV + DCV + RBV }\end{array}$ \\
\hline \multirow{2}{*}{2} & PeglFN + RBV & $\mathrm{SOF}+\mathrm{RBV}$ \\
\hline & $\mathrm{SOF}+\mathrm{RBV}$ & $\mathrm{VEL} / \mathrm{SOF}$ \\
\hline \multirow{3}{*}{3} & PeglFN + RBV & $\begin{array}{l}\text { SOF + PeglFN + RBV } \\
\text { SOF + RBV }\end{array}$ \\
\hline & $\begin{array}{l}\text { SOF + PeglFN + RBV } \\
\text { SOF + RBV }\end{array}$ & VEL/SOF \\
\hline & $\mathrm{SOF}+\mathrm{DCV}+\mathrm{RBV}$ & $\begin{array}{l}\text { F0-F3: more effective proposed therapies are awaited } \\
\text { F4: VEL/SOF }\end{array}$ \\
\hline \multirow[b]{2}{*}{4} & $\begin{array}{l}\text { PeglFN + RBV } \\
\text { SOF + PegIFN + RBV } \\
\text { SOF + RBV }\end{array}$ & $\begin{array}{l}\text { GZR/EBR } \pm \text { RBV } \\
\mathrm{LDV} / \mathrm{SOF} \pm \mathrm{RBV} \\
\mathrm{OBV} / \mathrm{PTV} / \mathrm{r}+\mathrm{RBV} \\
\text { VEL/SOF }\end{array}$ \\
\hline & $\begin{array}{l}\mathrm{GZR} / \mathrm{EBR} \pm \mathrm{RBV} \\
\mathrm{LDV} / \mathrm{SOF} \pm \mathrm{RBV} \\
\mathrm{OBV} / \mathrm{PTV} / \mathrm{r}+\mathrm{RBV} \\
\text { VEL/SOF }\end{array}$ & $\begin{array}{l}\text { F0-F3: more effective proposed therapies are awaited } \\
\text { F4: } \\
\text { SOF + GZR/EBR + RBV } \\
\text { SOF + OBV/PTV/r + DSV + RBV } \\
\text { SOF + SMV + DCV + RBV }\end{array}$ \\
\hline \multirow{3}{*}{5,6} & PeglFN + RBV & VEL/SOF \\
\hline & SOF + PeglFN + RBV & \\
\hline & SOF + RBV & \\
\hline
\end{tabular}

\section{Disclosure}

Waldemar Halota: Consultancy - AbbVie, BMS, Gilead, Janssen, Merck, Roche; Sponsored lectures AbbVie, BMS, Gilead, Janssen, Merck, Roche;

Robert Flisiak: Consultancy - AbbVie, BMS, Gilead, Janssen, Merck, Roche; Sponsored lectures: AbbVie, BMS, Gilead, Janssen, Merck, Roche;

Jacek Juszczyk: Consultancy - Merck; Sponsored lectures - Merck;

Piotr Małkowski: Consultancy - Gilead; Sponsored lectures - AbbVie, Gilead, BMS, MSD;

Małgorzata Pawłowska: Consultantcy - AbbVie, BMS, Gilead, Janssen, Merck, Roche; Sponsored lectures - AbbVie, BMS, Gilead, Janssen, Merck, Roche;

Krzysztof Simon: Consultancy - AbbVie, BMS, Gilead, Janssen, Merck, Roche, Bayer, Allergen; Sponsored lectures - AbbVie, BMS, Gilead, Janssen, Merck, Roche, Alfa Wasserman, Bayer;
Krzysztof Tomasiewicz: Consultancy - AbbVie, Alfa Wasserman, BMS, Gilead, Janssen, MSD, Roche; Sponsored lectures - AbbVie, Alfa Wasserman, BMS, Gilead, Janssen, MSD, Roche.

\section{References}

1. Wedemeyer H, Dore GJ, Ward JW. Estimates on HCV disease burden worldwide - filling the gaps. J Viral Hepatitis 2015; 22 (Suppl 1): 1-5.

2. Flisiak R, Halota W, Horban A, et al. Analysis of risk factors related to HCV infection in Poland. Eur J Gastroenterol Hepatol 2011; 23: 1213-1217.

3. Flisiak R, Halota W, Tomasiewicz K, et al. Forecasting the disease burden of chronic hepatitis $\mathrm{C}$ virus in Poland. Eur J Gastroenterol Hepatol 2015; 27: 70-76.

4. Flisiak R, Pogorzelska J, Berak H, et al. Prevalence of HCV genotypes in Poland - the EpiTer study. Clin Exp Hepatol 2016; 2: $144-148$.

5. Westbrook RH, Dusheiko G. Natural history of hepatitis C. J Hepatol 2014; 61: S58-68. 
6. Halota W, Flisiak R, Boroń-Kaczmarska A, et al.; Polish Group of HCV Experts. Recommendations for the treatment of hepatitis C issued by the Polish Group of HCV Experts - 2016. Clin Exp Hepatol 2016; 2: 27-33.

7. EASL Recommendations on Treatment of Hepatitis C 2016. J Hepatology 2017; 66: 153-194.

8. Basu P, Niraj JS, Nimy J, et al. Sofosbuvir and Ledipasvir versus Sofosbuvir and Simeprevir combination therapy in the management of acute hepatitis C: A randomized open label prospective clinical pilot study. SLAM C study. Interim data. AASLD 2015; 110318, ID: 1074

9. Jaroszewicz J, Flisiak-Jackiewicz M, Lebensztejn D, et al. Current drugs in early development for treating hepatitis $\mathrm{C}$ virusrelated hepatic fibrosis. Expert Opin Investig Drugs 2015; 24: 1229-1239.

10. www.hepatitisc.uw.edu/page/clinical-calculators/apri.

11. Kobayashi M, Suzuki F, Fujiyama S, et al. Sustained virologic response by direct antiviral agents reduces the incidence of hepatocellular carcinoma in patients with $\mathrm{HCV}$ infection. J Med Virol 2017; 89: 476-483.

12. ANRS collaborative study group on hepatocellular carcinoma (ANRS CO22 HEPATHER, CO12 CirVir and CO23 CUPILT cohorts). Lack of evidence of an effect of direct-acting antivirals on the recurrence of hepatocellular carcinoma: Data from three ANRS cohorts. J Hepatol 2016; 65: 734-740.

13. Nault JC, $m$ Colombo: Hepatocellular carcinoma and direct acting antiviral treatments: Controversy after revolution. J Hepatol 2016; 65: 741-747.

14. Calvaruso V et al. Early occurence of hepatocellular carcinoma (HCC) in patients with HCV cirrhosis treated with direct-acting antivirals (DAAs). EASL HCC Summit, Geneva, 2-5 February 2017. COO3.

15. Cavaletto $\mathrm{L}$ et al. Comparison between de-novo occurence and recurrence of hepatocellular carcinoma (HCC) after direct-acting antivirals (DAAs) in cirrhotic patients with hepatitis C: A real-life cohort study. EASL HCC Summit, Geneva, 2-5 February 2017. PIO.05.

16. Kolly $\mathrm{P}$ et al. Hepatocellular carcinoma after direct antiviral agent treatment: A European Multicenter study. EASL HCC Summit, Geneva, 2-5 February 2017. PII.09-YI.

17. Gheorghe L et al. Alpha fetoprotein - a useful for follow-up of interferon-free treated cirrhotic patients with de novo hepatocellular carcinoma after SVR. EASL HCC Summit, Geneva, 2-5 February 2017. PI2.09.

18. Castano A et al. Alpha fetoprotein (AFP) levels before and after sustained virological response with direct-acting antivirals (DAAs) in patients with liver cirrhosis due to hepatitis $\mathrm{C}$ virus (HCV). EASL HCC Summit, Geneva, 2-5 February 2017. PI3.04-YI.

19. Conti F, Buonfiglioli F, Scuteri A, et al. Early occurence and recurrence of hepatocellular carcinoma in HCV-related cirrhosis treated direct-acting antivirals. J Hepatol 2016; 65: 727-733.

20. Reig M, Mariño Z, Perelló C, et al. Unexpected high rate of early tumor recurrence in patients with HCV-related HCC undergoing interferon-free therapy. J Hepatol 2016; 65: 719-726.

21. Wasiak D, Małkowski P. Wytyczne leczenia raka wątrobowokomórkowego (HCC). Med Sci Mon Rev Hepatology 2013; 13: 112-115.

22. Bersoff-Matcha SJ, Cao K, Jason M, et al. Hepatitis B Reactivation in Patients Receiving Interferon-Free Direct Acting Antiviral Agents for Chronic Hepatitis C Virus Infection. AASLD 2016 Nov 11-15 Boston.

23. AASLD/IDSA Recomendations for Testing, Managing, and Treating Hepatitis C updated, Sep 2016.
24. Ende AR, Kim NH, Yeh MM, et al. Fulminant hepatitis B reactivation leading to liver transplantation in a patient with chronic hepatitis $C$ treated with simeprevir and sofosbuvir: a case report. J Med Case Rep 2015; 9: 164.

25. Badri P, Dutta S, Coakley E. Pharmacokinetics and dose recommendations for cyclosporine and tacrolimus when coadministered with ABT-450, ombitasvir, and dasabuvir. Am J Transplant 2015; 15: 1313-1322.

26. www.hep-druginteractions.org.

27. Flisiak R, Janczewska E, Wawrzynowicz-Syczewska M, et al. Real-world effectiveness and safety of ombitasvir/paritaprevir/ ritonavir+dasabuvir+ribavirin in hepatitis C: AMBER study. Aliment Pharmacol Ther 2016; 44: 946-956.

28. Juszczyk J. Hepatitis C, patogeneza i terapia. Termedia, Poznań 2016.

29. Calleja JL, Crespo J, Rincón D, et al. Effectiveness, safety and clinical outcomes of direct-acting antiviral therapy in HCV genotype 1 infection: results from a Spanish real world cohort. J Hepatol 2017; doi: 10.1016/j.jhep.2017.01.028. [Epub ahead of print].

30. Viekirax, Charakterystyka Produktu Leczniczego.

31. Exviera, Charakterystyka Produktu Leczniczego.

32. Harvoni, Charakterystyka Produktu Leczniczego.

33. Flisiak R, Pogorzelska J, Berak H, et al. Efficacy of HCV treatment in Poland at the turn of the interferon era - the EpiTer study. Clin Exp Hepatol 2016; 2: 138-143.

34. Flisiak R, Łucejko M, Mazur W, et al. Effectiveness and safety of ledipasvir/sofosbuvir \pm ribavirin in the treatment of HCV infection: The real-world HARVEST study. Adv Med Sci 2017 [In press].

35. Daklinza, Charakterystyka Produktu Leczniczego.

36. Kumada H, Suzuki Y, Ikeda K i wsp. Daclatasvir plus Asunaprevir for chronic HCV genotype $1 \mathrm{~b}$ infection. Hepatology 2014; 59: 2083-2091.

37. Manns M, Pol S, Jacobson IM, et al. All-oral daclatasvir plus asunaprevir for hepatitis $\mathrm{C}$ virus genotype $1 \mathrm{~b}$ : a multinational, phase 3 , multicohort study. Lancet 2014; 384: 1597-1605.

38. Zepatier, Charakterystyka Produktu Leczniczego.

39. Epclusa, Charakterystyka Produktu Leczniczego.

40. Pegasys, Charakterystyka Produktu Leczniczego.

41. Pegintron, Charakterystyka Produktu Leczniczego.

42. Sovaldi, Charakterystyka Produktu Leczniczego.

43. Cornberg M, Petersen J, Schober A, et al. Real-world use, effectiveness and safety of anti-viral treatment in chronic hepatitis $\mathrm{C}$ genotype 3 infection. Aliment Pharmacol Ther 2017; 45: 688-700. 
